\title{
Artificial intelligence and collective intelligence: the emergence of a new field
}

\author{
Geoff Mulgan ${ }^{1}$
}

Published online: 20 September 2018

(c) Springer-Verlag London Ltd., part of Springer Nature 2018

The great majority of debate about artificial intelligence (AI) starts with the capabilities of existing or emergent technologies-machine learning, deep learning, computer vision, natural language processing and so on-and then asks where these might be used. But an alternative approach turns the question on its head and asks how institutions or systems could be more intelligent. The spread of intelligent machines often coincides with less intelligence at a systems level. How should 'thought' happen at a large scale, involving many people and often many machines, to better solve problems? In particular, how could AI be used to address complex social problems?

We see great potential in AI, if handled well, but we also believe that many of the biggest gains will come from better approaches to combining human and machine intelligence, in particular harnessing the intelligence of groups.

That is why Nesta is creating a new centre (https://www. nesta.org.uk/project/centre-collective-intelligence-design/) to focus on the practical skills needed to design intelligence well, looking first at the problems and challenges, rather than technologies. How could a city better manage its labour markets or air quality? How could cancer make the most of both medical knowledge and patient experience? How could school systems mobilise the insights of teachers as well as data and algorithms to better teach young people?

\section{The theory}

Some of the answers depend on better theory. My book 'Big Mind; how collective intelligence can change our world' (from Princeton University Press) sets out some of the building blocks for collective intelligence. I break intelligence

\section{Geoff Mulgan}

geoff.mulgan@ nesta.org.uk

1 CEO Nesta, 58 Victoria Embankment, London EC4Y 0DS, UK down into component parts - observation, analysis, memory, creativity, judgement and wisdom - and show how very different methods are needed for these, and very different combinations of human and machine capabilities.

I also show the organisational conditions for intelligence at scale, such as what I call the creation of an 'autonomous commons', a space for thought and criticism at once removed from interests and egos, and a good balance between the components of intelligence. This theory is then applied to particular fields-like democracy-each of which can be rethought as an expression of collective intelligence.

However, this brings us to a challenge-there is an absence of institutions devoted to what we call 'intelligence design'. There are pockets of deep skill—some within commercial firms like Amazon and Google—but very little of this has been codified or shared more widely. As a result, and despite the spread of smart technologies, many of the systems we depend on most are much less ingenious than they could be.

\section{Designing collective intelligence-the role of humans and machines}

I have argued in the past that progressing collective intelligence is in many ways humanity's grandest challenge since understanding how we think and act together is integral to solving other challenges. Rapid advances in technology mean that we have more opportunities than ever before to progress collective intelligence and many organisations are experimenting with new ways to harness the insights of large groups, in fields ranging from citizen science to democracy.

Some of our projects, such as our study of digital social innovation in Europe, have shown how digital platforms offer the potential to assemble people and knowledge as never before. In Europe alone there are thousands of initiatives using digital platforms to mobilise large groups of people and their knowledge to address challenges in all areas 
of society. Some of these make use of rapid advances in artificial intelligence and machine learning that have enabled new ways of capturing, analysing and learning from large amounts of complex data.

So far, however, the vast majority of practice, policy and research has given little attention to the potential for innovation when human and machine intelligence are combined. There is an emerging academic discipline of collective intelligence and a field of promising practice, but it remains tiny by comparison with artificial intelligence.

\section{The centre for collective intelligence design}

The centre, which we will launch this autumn, will grow expertise in both the understanding and practice of collective intelligence, mobilising human intelligence at scale and combining it with data, $\mathrm{AI}$ and other resources.

The centre will build on Nesta's existing work in this area and insights from many authors and research centres around the world (such as the Collective Intelligence Unit at Copenhagen Business School, the MIT Center for Collective Intelligence, The MIT Intelligence Quest and The Alan Turing Institute to name a few). We will be drawing on insights from many organisations-ranging from businesses to NGOs.

Our aim is to help build a field that combines the best of neighbouring fields, while helping mobilise intelligence to solve practical problems. We are convinced that every organisation can work more successfully if it taps into a bigger mind-mobilising more brains and computers to help it. But doing that requires careful design, curation and orchestration. It is not enough just to mobilise the crowd (since crowds are all too capable of being foolish, prejudiced and malign) or to hope that brilliant ideas will emerge naturally.
Thought requires work- to observe, analyse, create, remember and judge.

Although there is huge investment in artificial intelligence, there has been much less investment in collective intelligence. That is one reason why we have also seen little progress in how intelligently our most important systems work-democracy and politics, business and the economy. We are surrounded by institutions packed with individual intelligence that nevertheless often display collective stupidity.

Many of the examples I look at in my book are quite old-like the Oxford English Dictionary which mobilised tens of thousands of volunteers in the nineteenth century, or NASA's Apollo programme which at its height employed over half a million people in more than 20,000 organisations. The tools at our disposal are radically different-and more powerful than ever before. We can learn much from looking at past examples like the Cybersyn project in Chile in the early 1970s that tried to create a collective intelligence for the economy.

It is easy to be depressed by the many examples of collective stupidity around us. But my instinct is to be optimistic that we will figure out how to make the smart machines we have created serve us well and that we could be on the cusp of a dramatic enhancement of our shared intelligence.

Curmudgeon Corner Curmudgeon Corner is a short opinionated column on trends in technology, arts, science and society, commenting on issues of concern to the research community and wider society. Whilst the drive for super-human intelligence promotes potential benefits to wider society, it also raises deep concerns of existential risk, thereby highlighting the need for an ongoing conversation between technology and society. At the core of Curmudgeon concern is the question: What is it to be human in the age of the AI machine? -Editor. 